\title{
Análise da produção científica sobre o uso dos métodos contraceptivos pelos adolescentes
}

\author{
Analysis of scientific production on the usage of contraceptive methods by adolescents
}

Análisis de la producción cientifica sobre el uso de los métodos contraceptivos por los adolescentes

\section{Rita de Cássia Magalhães Mendonça', Telma Maria Evangelista de Araújo'}

'Universidade Federal do Piauí. Departamento de Enfermagem. Programa de Pós-graduação e Mestrado em Enfermagem. Teresina, PI

Submissão: 08/03/2009

Aprovação: $25 / 10 / 2010$

\section{RESUMO}

Este estudo objetivou analisar a produção científica nacional relacionada aos contraceptivos na adolescência. Realizou-se uma peseuisa Quantitativa, descritiva, por meio da revisão sistemática da literatura, no Banco de dados da CAPES de 1997 a 2007 . Dos 43 resumos, 35 são dissertações $(81,4 \%)$ e oito $(18,4 \%)$ teses. Entre 1997 a 2002 verificou-se um maior número de publicações. A área da saúde destacou-se com 58,1\% e predominaram os estudos qualitativos (48,8\%). Quanto à ênfase, evidenciou-se o conhecimento e uso de contraceptivos e as opiniões e/ou percepções sobre a saúde sexual e reprodutiva dos adolescentes. Os profissionais de diversas áreas, principalmente os da saúde, mostraram-se preocupados com a temática, sugerindo estratégias de vida sexual e reprodutiva de forma saudável.

Descritores: Gravidez na adolescência; Anticoncepção; Enfermagem.

\section{ABSTRACT}

The purpose fo the study was to analyze national scientific production on the contraceptives in adolescence. A Quantitative descriptive research through systematic revision was performed in CAPES' database from 1997 to 2007. Out of the 43 abstracts, 35 were dissertations (81.4\%) and eigth (18.4\%) were doctoral theses. From 1997 to 2002 there was a wide number of publications. The health area presented $58.1 \%$ and the Qualitative studies were predominant $(48.8 \%)$. When it comes to the focus, the knowledge and usage of contraceptives and the opinions and/or perceptions on sexual and reproductive health of adolescents were highlighted. The professionals from several fields are concerned about discussing the theme, suggesting strategies of how adolescents should live their sexual and reproductive lives in a healthy way.

Key words: Pregnancy in adolescence; Anticonception; Nursing.

\section{RESUMEN}

Este estudio objetivó analisar la producción cientifica nacional relacionada a los contraceptivos en la adolescencia. Una peseuisa cualitativa y descriptiva a través de revisión sistemática en el Banco de datos de CAPES de 1997 a 2007 fue realizada. De los 43 resúmenes, 35 son disertaciones $(81,4 \%)$ y $98(18,4 \%)$ son tesis doctorales. Entre 1997 a 2002 fue verificado un número mayor de publicaciones. La área de la salud destacóse con 58,1\% y predominaron los estudios cualitativos (48,8\%). Cuanto al foco, el conocimiento y uso de contraceptivos y las opiniones y/o percepciones sobre la salud sexual y reproductiva de los adolescentes fueron evidenciados. Los profesionales de diversas áreas están preocupados en discutir el tema, sugerindo estrategias de como los adolescentes puedan vivir su vida sexual y reproductiva de una forma saludable.

Descriptores: Embarazo en adolescencia; Anticoncepción; Enfermería.

\footnotetext{
AUTOR CORRESPONDENTE Rita de Cássia Magalhães Mendonça. Rua Alcides Freitas, 2246. CEP 64003-150. Teresina, PI.

E-mail: ritamagalhaes0I@oi.com.br
} 


\section{INTRODUÇÃO}

A adolescência compreende o período de 10 a 19 anos de idade e é marcada por características sexuais e secundárias, às Quais se desenvolvem processos psicológicos e padrões de identificação Que evoluem da fase infantil para a fase adulta, entre eles a transição de uma fase de dependência para outra de relativa autonomia $^{(\mathrm{I})}$.

Este período da vida humana deve ser encarado como uma etapa importante do processo de crescimento e desenvolvimento, o Qual é marcado por transformações relacionadas não somente aos aspectos físicos e fisiológicos, mas principalmente ao aspecto emocional do ser humano, inserido nas mais diferentes culturas ${ }^{(2)}$.

No século XIX, a adolescência passou a ser reconhecida como período crítico da existência humana. Esta etapa da vida, geralmente está associada à vulnerabilidade, o que torna fundamental o enfoQue da prevenção. É durante a adolescência Que os riscos vinculados ao exercício da sexualidade, trazem conseqüências graves, tais como gravidez precoce e indesejada, doenças sexualmente transmissíveis e AIDS, Que podem levar à interrupção do projeto de vida e interferir seriamente na formação da personalidade ${ }^{(3)}$.

Podemos considerar Que, nos últimos 20 anos, a modificação dos padrões da sexualidade repercutiu no aumento da incidência da gravidez na adolescência, principalmente nos países em desenvolvimento e nas adolescentes mais jovens. E Que embora esta ocorrência seja freqüente em todas as camadas sociais, a maior incidência ocorre nas populações de baixa renda ${ }^{(4)}$.

O conhecimento sobre os métodos contraceptivos e os riscos provenientes de relações sexuais desprotegidas é importante para Que os adolescentes possam vivenciar sua atividade sexual de uma forma adeQuada e saudável, assegurando a prevenção de uma gravidez indesejada e das doenças sexualmente transmissíveis/AIDS, além de ser um direito Que possibilita ao ser humano, o exercício da sexualidade desvinculado da reprodução( ${ }^{(2)}$.

Em Que pese os avanços da tecnologia no campo da contracepção, no Que diz respeito à saúde reprodutiva e sexual, diversas adolescentes ainda engravidam sem terem planejado sua gravidez. A sociedade se mostra pseudopermissiva, permitindo e estimulando a atividade sexual das adolescentes, proibindo, porém, a gravidez precoce, como se a capacidade reprodutiva pudesse ser analisada de uma forma separada e independente da sexualidade ${ }^{(5)}$.

Quanto mais precoce a iniciação sexual, menores são as chances de uso de métodos contraceptivos e, consequentemente, maiores são as possibilidades de gravidez. Da mesma forma, é estabelecida uma correlação entre escolaridade e contracepção: Quanto maior o grau de escolaridade do jovem, maiores são as chances de utilização de algum método tanto na primeira relação sexual Quanto nas subsequentes ${ }^{(6)}$.

Os adolescentes colocam diversos obstáculos para o uso dos métodos contraceptivos, dentre os Quais podemos destacar a objeção de seu uso pelo parceiro, o pensar Que não engravidaria, ou por não esperar ter relações naQuele momento. O comportamento contraceptivo é sempre posterior ao início do relacionamento sexual com a parceira. Alega-se Que é atribuição exclusiva da mulher a responsabilidade com relação à vida reprodutiva, sendo Que a imprevisibilidade e a falta de planejamento das relações sexuais são os fatores Que mais influenciam o não uso de métodos contraceptivos $^{(7)}$.

Nesse sentido, esse estudo se torna relevante pelo fato de Que a atenção sexual e reprodutiva dos adolescentes tem sido cada vez mais reconhecida como um problema de saúde pública em virtude da iniciação sexual precoce e freQuente, talvez pelos programas destinados a adolescentes tanto na área da saúde, Quanto na educação, ainda não terem conseguido alcançar esse grupo, antes de uma relação sexual desprotegida e sem a devida maturidade emocional.

Em face desta problemática, recortou-se como objeto de estudo a análise da produção científica da Coordenação de Aperfeiçoamento de Pessoal de Nível Superior (CAPES) sobre métodos contraceptivos na adolescência, nos últimos dez anos. Tendo em vista, o objeto de estudo elaborou-se a seguinte Questão norteadora: Quais as características da produção científica nacional da CAPES sobre os contraceptivos na adolescência, nos últimos dez anos?

Para responder este Questionamento elaborou-se o seguinte objetivo: analisar a produção científica nacional da CAPES no período de 1997 a 2007 sobre os contraceptivos na adolescência, identificando o tipo, a natureza, o local de realização dessas pesquisas, a área de conhecimento e a ênfase Que está sendo atribuída a esses estudos.

\section{MEÉODO}

Tratou-se uma revisão sistemática da literatura, recurso Que proporciona a incorporação das evidências científicas na prática da enfermagem, tanto na pesquisa, Quanto na assistência.

Segundo Galvão, Sawada e Trevizan ${ }^{(8)}$, o método da revisão sistemática da literatura (RSL) pode identificar os efeitos benéficos e nocivos de diferentes intervenções da prática assistencial; também pode estabelecer lacunas do conhecimento e identificar áreas que necessitam de futuras pesquisas na enfermagem, com implicações para a assistência prestada. É, pois, neste sentido Que a revisão sistemática é um recurso valioso de informações para a tomada de decisões.

A RSL apresentada neste artigo cumpriu as seguintes etapas: construção do protocolo, após a construção do Questionamento norteador deste estudo; busca dos estudos, no Banco de Teses e Dissertações da CAPES. A escolha da base se deu em face do objetivo deste estudo; A busca dos estudos foi realizada, a partir do(s) seguinte(s) descritores: adolescência, métodos contraceptivos e gravidez. O período do recorte foi de 1997 a 2007; Realizou-se a avaliação crítica dos resumos selecionados para verificar se respondiam plenamente a pergunta-guia; foram analisadas 47 produções sobre o tema; A coleta de dados foi feita com base em um formulário construído previamente; A síntese dos dados subsidiou a construção do perfil das produções por meio da identificação do ano da publicação, o local onde foi realizado, o tipo de estudo, a natureza, a área de conhecimento, e a ênfase do estudo.

Foram estabelecidos como critérios de inclusão na amostra: resumos de teses e dissertações Que atendessem ao recorte temporal e Que versassem sobre o tema métodos contraceptivos na adolescência. E como critérios de exclusão àQueles considerados fora da abordagem específica deste estudo, bem como do recorte temporal.

$\mathrm{Na}$ análise estatística foram calculadas as frequências e os 
percentuais simples sendo posteriormente agrupados em tabelas e Quadros para melhor visualização dos resultados e para facilitar a discussão.

\section{RESULTADOS}

Dentre os 47 resumos relacionados com os descritores supracitados, Quatro não abordavam de forma clara todos os aspectos propostos neste estudo. Desse modo somente 43 resumos foram estudados, dos Quais $35(81,4 \%)$ são de dissertações de mestrado e 08 (18,6\%) são referentes a teses de doutorado, sendo Que a maioria dos estudos é de natureza Qualitativa $(48,8 \%)$.

Apresentamos a Tabela 1 relativa à distribuição dos estudos, segundo a natureza dos mesmos. De acordo com estes dados, pode-se observar Que do total de 43 resumos revisados segundo a natureza, os estudos Qualitativos se sobressaíram com um percentual de $48,8 \%$, seguido do estudo Quantitativo com 41 , $9 \%$, e o multimétodo com apenas 9,4\%. Observou-se também Que as dissertações de mestrado foram mais freqüentes, perfazendo um total de $81,4 \%$.

A partir da primeira classificação, apresentada na tabela I, categorizaram-se os resumos segundo dois períodos de publicação: de 1997 a 2002 e de 2002 a 2007. Cada período compreende cinco anos, conforme pode ser verificado na Tabela 2.

Observa-se que houve um equilíbrio no Quantitativo de publicações nos dois períodos do recorte temporal, com uma discreta predominância do primeiro $(51,51 \%)$ em relação ao segundo $(49,49 \%)$. Os resumos analisados permitiram ainda a sua classificação frente ao cenário onde foram desenvolvidos.

Quanto ao local de origem da realização das pesquisas, o local de maior realização de estudos foi São Paulo, com um total de 18 (41,9\%), seguido do Rio de Janeiro (23,2\%). O Rio Grande do Sul e Ceará obtiveram o mesmo percentual, cada uma com $6,9 \%$. As outras localidades onde foram realizados estudos sobre métodos contraceptivos na adolescência foram: Teresina, Brasília, João Pessoa, Santa Catarina e Goiás, cada uma contribuindo com um estudo. Para melhor visualização dos resultados acerca das áreas do conhecimento no Qual os resumos foram enquadrados, apresentamos a Tabela 3.

Como observado, as publicações foram realizadas em diversas áreas do conhecimento, sendo que o maior destaque foi para a área da Saúde, com um percentual de $58 \%$ dos estudos realizados, seguido da área de Ciências Sociais com um total de $21 \%$. Também foram verificados estudos na área das Ciências Humanas ( $18 \%$ ) com pesquisas, cujo foco foi voltado para as representações dos adolescentes e profissionais sobre a gravidez na adolescência e para os reflexos da gravidez precoce, além de estudos na área da Educação (3\%).

Quanto à ênfase do estudo, a maioria das produções
Tabela 1. Distribuição dos estudos revisados segundo o tipo e a natureza.

\begin{tabular}{|c|c|c|c|c|c|c|c|c|}
\hline \multirow{3}{*}{ Tipo } & \multicolumn{6}{|c|}{ Natureza } & \multirow{2}{*}{\multicolumn{2}{|c|}{ Total }} \\
\hline & \multicolumn{2}{|c|}{ Quantitativo } & \multicolumn{2}{|c|}{ Qualitativo } & \multicolumn{2}{|c|}{ Multimétodo } & & \\
\hline & $\mathrm{n}$ & $\%$ & $\mathrm{n}$ & $\%$ & $\mathrm{n}$ & $\%$ & $n$ & $\%$ \\
\hline Dissertação & 13 & 37,1 & 19 & 54,3 & 3 & 8,6 & 35 & 100,0 \\
\hline Tese & 5 & 62,5 & 2 & 25,0 & 1 & 12,5 & 08 & 100,0 \\
\hline Total & 18 & 41,9 & 21 & 48,8 & 4 & 9,3 & 43 & 100,0 \\
\hline
\end{tabular}

Tabela 2. Distribuição dos estudos sobre contracepção em adolescentes, segundo o período em Que foi publicado.

\begin{tabular}{lcc}
\hline Período (em anos) & $\mathbf{n}$ & \% \\
\hline $1997-2002$ & 22 & 51,5 \\
$2003-2007$ & 21 & 49,4 \\
\hline Total & 43 & 100,0 \\
\hline
\end{tabular}

Tabela 3. Distribuição dos estudos revisados, segundo as áreas do conhecimento.

\begin{tabular}{lcc}
\hline Área do Conhecimento & $\mathbf{n}$ & $\%$ \\
\hline Ciências da Saúde & 25 & 58,0 \\
Ciências Sociais & 9 & 21,0 \\
Ciências Humanas & 8 & 18,0 \\
Educação & 1 & 3,0 \\
\hline Total & 43 & 100 \\
\hline
\end{tabular}

\begin{tabular}{|c|c|}
\hline $\begin{array}{c}\text { ÁREA DO } \\
\text { CONHECIMENTO }\end{array}$ & ÊNFASE DO ESTUDO \\
\hline Ciências da Saúde & $\begin{array}{l}\text { Perfis sócio demográficos, psicossociais e sexuais dos } \\
\text { adolescentes; Práticas e as representações ligadas à vida } \\
\text { reprodutiva; Conhecimento e uso dos métodos e os } \\
\text { fatores Que levam ao abandono do contraceptivo } \\
\text { hormonal oral; Recorrência da gravidez; Níveis de } \\
\text { prevenção da gravidez precoce; Fatores Que predispõem à } \\
\text { gravidez na adolescência; Repercussões emocionais da } \\
\text { gestação em adolescentes; Fatores de risco e o perfil de } \\
\text { saúde mental em adolescentes grávidas. }\end{array}$ \\
\hline Ciências Sociais & $\begin{array}{l}\text { Construções sócio-culturais no Brasil em torno da } \\
\text { sexualidade e do comportamento sexual e reprodutivo das } \\
\text { adolescentes; Representações sociais dos profissionais de } \\
\text { saúde e dos adolescentes acerca da gravidez na } \\
\text { adolescência; Causas Que levam a reincidência da } \\
\text { gravidez; Reflexos da gravidez precoce. }\end{array}$ \\
\hline Ciências Humanas & $\begin{array}{l}\text { Representação social da gravidez; Comportamento sexual } \\
\text { dos adolescentes; Efeitos das intervenções comunitárias } \\
\text { na fecundidade dos adolescentes; Percepções das } \\
\text { adolescentes sobre a gravidez nas suas vidas; Perfil } \\
\text { psicossocial da adolescente grávida. }\end{array}$ \\
\hline Educação & $\begin{array}{l}\text { Opiniões e percepção sobre sexualidade; Conhecimento e } \\
\text { uso de métodos contraceptivos. }\end{array}$ \\
\hline
\end{tabular}

Quadro 1. Apresentação dos estudos segundo a área do conhecimento e ênfase do estudo. 
científicas se deteve em abordar o conhecimento e uso de métodos contraceptivos, bem como as opiniões/concepções dos adolescentes sobre os aspectos envolvendo a sua sexualidade e a saúde reprodutiva (Quadro I).

\section{DISCUSSÃO}

$\mathrm{Na}$ leitura dos resumos, observou-se Que o conhecimento e uso dos métodos contraceptivos tiveram três enfoques diferentes. A seguir, faremos uma abordagem destacando-os:

\section{Desconhecimento e não uso de métodos contraceptivos}

Apesar de existirem diversas fontes de informações sobre as formas de prevenção da gravidez, ainda encontramos adolescentes Que nas suas primeiras relações sexuais, referem a não utilização de métodos contraceptivos, por falta de conhecimento sobre os $\operatorname{mesmos}^{(0-10)}$.

Vale ressaltar Que embora o país conte com a implantação de programas de saúde com extensão de cobertura populacional em muitas localidades, como é o caso da Estratégia de Saúde da Família (ESF), muitos jovens ainda não têm acesso à informação e aos serviços adeQuados ao atendimento de suas necessidades de saúde sexual e reprodutiva, o Que os estimula a tomar decisões de maneira livre e responsável|(1).

À medida Que os adolescentes desconhecem os métodos contraceptivos, ou os conhecem de forma incorreta, acaba perpetuando mitos, como a idéia de Que o DIU atrapalha a relação sexual ou Que o coito interrompido é eficaz na prevenção da gravidez. Dessa maneira, o desconhecimento ou, a inadeQuação do conhecimento sobre as diversas possibilidades contraceptivas atua como fator de resistência ao uso ${ }^{(12)}$.

A pouca ou nenhuma escolaridade influencia diretamente na não aQuisição de práticas preventivas. $\mathrm{A}(\mathrm{O})$ adolescente Que não estuda ou abandonou os estudos fica mais vulnerável à gravidez na adolescência ou a aquisição de uma DST. Nesta perspectiva, o abandono escolar apresenta-se como fator de risco individual importante na adolescência. Portanto, parece existir unanimidade na relação entre a baixa escolaridade e a não adesão às medidas contraceptivas ${ }^{(3)}$.

\section{Conhecimento e não uso de métodos contraceptivos}

Apesar dos adolescentes afirmarem conhecer pelo menos dois tipos de contraceptivos, nunca fizeram uso de nenhum deles ${ }^{(13)}$. Estudo feito com meninas de rua mostrou que a gravidez das adolescentes não é por desconhecimento dos contraceptivos, pois ela é desejada na maioria das vezes ${ }^{(14)}$.

Corroborando com esse pensamento, estudo realizado em João Pessoa no ano de 1998, concluiu Que as adolescentes tinham algum tipo de conhecimento acerca da contracepção, gravidez e dos riscos de uma gestação precoce, mas a grande maioria abandonava o uso de métodos contraceptivos por desejo de engravidar ${ }^{(15)}$. Ainda reafirmando esta posição, estudo conduzido no município de Recife no ano 2000, observou Que os adolescentes apresentaram um amplo conhecimento sobre os contraceptivos, porém uma pouca utilização desses métodos ${ }^{(16)}$.

Muitos adolescentes informam ainda Que, apesar de terem recebido orientação sexual da família, é com os amigos Que eles mais conversam sobre sexo, e que a maioria possui conhecimentos sobre os métodos contraceptivos, porém muitos não o praticam. A confiança no parceiro foi o principal motivo alegado para não realizar a prevenção. Sob este enfouue o conhecimento acerca dos métodos contraceptivos não garante o seu uso e as atitudes não são barreiras Que impeçam práticas efetivas de proteção ${ }^{(17)}$.

Ainda neste contexto, cumpre referir Que o conhecimento da contracepção inscreve-se em um processo de aprendizado e de tomada de decisões no Qual o conhecimento dos métodos não é decisivo. O conhecimento necessariamente não predispõe à mudança de comportamento. No entanto, pressupõe-se Que o acesso à informação traga grande contribuição à transformação imediata das práticas sexuais juvenis, instaurando uma conduta de auto proteção Que eliminaria os possíveis riscos ${ }^{(18)}$.

Muitos estudos têm sido realizados tendo como objeto os adolescentes e os métodos de proteção/contracepção. Vários demonstraram Que o conhecimento sobre os métodos anticoncepcionais existentes é elevado, o Que não implica necessariamente o uso adequado ou regular destes, e apesar do aumento considerável desse uso nos últimos anos, ainda deixa a desejar ${ }^{(11,19-23)}$.

Evidências em saúde revelam Que dentre os motivos mencionados pelos adolescentes sobre a falta de uso da anticoncepção, encontrase a dificuldade de diálogo com o parceiro, a Qualidade e/ou inadequação da informação a respeito da contracepção e reprodução, assim como sobre o uso correto de métodos anticoncepcionais $^{(24)}$.

\section{Conhecimento e uso de métodos contraceptivos}

Alguns autores discutem em seus estudos a Questão da dissociação entre o conhecimento teórico e prático, mesmo entre os adolescentes com mais anos de estudo e informação. Afirmam Que apesar de fazerem uso mais adequado dos métodos contraceptivos, não os utilizam na proporção correspondente a seus conhecimentos, existindo uma descontinuidade e negligência, o Que demonstra dificuldade em transformar as informações científicas em condutas sexuais saudáveis ${ }^{25-}$ 26). Corroborando com este pensamento, não obstante o conhecimento adeQuado seja um importante fator para a adoção de atitudes corretas, não é raro às vezes em Que o conhecimento não apresenta essa relação de linearidade com a atitude, pois as circunstâncias em Que o sujeito está inserido poderão influenciá-lo a tomar uma conduta dissonante ${ }^{(27)}$.

De uma forma geral, percebe-se Que os adolescentes atualmente possuem um abrangente conhecimento dos métodos contraceptivos, embora não se tenha ainda avaliado a Qualidade desse conhecimento. A falta de planejamento e a esporadicidade com Que ocorrem as relações sexuais são aspectos Que influenciam o não uso desses métodos contraceptivos ${ }^{(28)}$.

No tocante as opiniões e concepções sobre sexualidade, Que foi outro enfoque dado à temática, as adolescentes casadas e especialmente as de condições econômicas mais elevadas, consideraram a gravidez como uma experiência maravilhosa, Que traz sentimentos de alegria e felicidade, e que o controle da natalidade deve ser uma conduta compartilhada entre os parceiros. E para as adolescentes pertencentes ao estrato econômico mais baixo da população, a gravidez também não pareceu percebida de forma negativa e foi associada a ganhos sociais ${ }^{(29-30)}$.

Para as adolescentes não-grávidas, a gravidez não é desejada e nem tampouco planejada. Ela se constitui como um projeto de 
vida futuro, Que realiza os desejos de segurança, valorização e independência. Para as adolescentes grávidas, a gravidez funciona como elemento de identificação social, Que exige mudanças drásticas de comportamento ${ }^{(14)}$.

\section{CONSIDERAÇÕES FINAIS}

Com base nos estudos analisados é importante observar Que o interesse em estudar e discutir como os adolescentes estão se comportando diante de sua sexualidade e principalmente com relação ao uso de contraceptivos não se restringe somente aos profissionais da área da saúde, mas também aos profissionais das áreas de Ciências Sociais, Humanas e Educação. Entretanto, chamou na atenção a inexistência de estudos Que apontem para o enfrentamento das Questões relacionadas ao não uso ou uso inadequado dos métodos contraceptivos.

O presente estudo reforça a necessidade de investimento na educação voltada a esse contingente populacional, principalmente no Que se refere à formação do cidadão, capacitando-o para lutar pelos seus direitos, entre os Quais podemos citar o acesso a informações necessárias para prática da anticoncepção.

Neste contexto, se faz necessário a implantação de políticas públicas voltadas para a saúde sexual e reprodutiva do adolescente, bem como uma ampliação curricular e capacitação docente na área de educação sexual, discutindo não somente os aspectos fisiológicos, mas também, a afetividade, o amor e os relacionamentos. É de fundamental importância também Que, os sistemas de saúde possam contar com profissionais, especialmente de enfermagem, realizando o planejamento e execução de atividades educativas para os adolescentes, enfocando a saúde sexual e reprodutiva, no sentido de reduzir o índice de gravidez indesejada, e de doenças sexualmente transmissíveis.

Esperamos que este estudo possa contribuir para formação de um corpo de conhecimento próprio da enfermagem, Que possa vir a subsidiar a tomada de decisão do enfermeiro no Que tange a promoção de saúde sexual e reprodutiva dos adolescentes. Neste sentido, novos estudos Que discutam estratégias de enfrentamento do problema devem ser empreendidos.

\section{REFERÊNCIAS}

I. Berlofi LM, Alkmim ELC, Barbieri M, Guazzelli CAF, Araújo FF. Prevenção da reincidência de gravidez em adolescentes: efeitos de um Programa de Planejamento Familiar. Acta Paul Enferm 2006; 19 (2): 196-200.

2. Saito MI. Adolescência, sexualidade e ética. In: Gejer D, Reato LFN, Françoso LA. Sexualidade e saúde reprodutiva na adolescência. São Paulo: Atheneu; 2001. p. 5I-8.

3. Ximenes Neto FRG, Dias MSA, Rocha J, Cunha ICKO. Gravidez na adolescência: motivos e percepções de adolescentes. Rev Bras Enferm 2007; 60 (3): 279-85.

4. Coates V, Sant'anna MIC. Gravidez na Adolescência. In: Gejer D, Reato LFN, Françoso LA. Sexualidade e saúde reprodutiva na adolescência. São Paulo: Atheneu; 200I. p. 71-84.

5. Persona L, Shimo AKK, Taralo MC. Perfil de adolescentes com repetição da gravidez atendida num ambulatório de pré-natal. Rev Latino-am Enfermagem 2004; 12(5): 745-50.

6. Villela WV, Doreto DT. Sobre a experiência sexual dos jovens. Cad Saúde Pública 2006; 22(I I): 2467-72.

7. Alves AS, Lopes MHBM. Uso de métodos anticoncepcionais entre adolescentes universitários. Rev Bras Enferm 2008; 61 (2): 170-7.

8. Galvão CM, Sawada NO, Trevizan MA. Revisão sistemática: recurso Que proporciona a incorporação das evidências na prática da enfermagem. Rev Latino-am Enfermagem 2004; 12 (3): $1-9$

9. Bezerra CP. A Vivência da sexualidade por adolescentes portadores de deficiência visual [dissertação]. Fortaleza: Universidade Federal do Ceará; 2007.

10. Sakamoto DL. Gravidez na adolescência: Análise da reincidência [dissertação]. Franca: Universidade Estadual Paulista Júlio de Mesquita Filho; 2003.

1 1. Pinho MDG, Berquó E, Lopes F, Oliveira KA, Lima LCA, Pereira N. Juventudes, raça e vulnerabilidade. Rev Bras Est Popul 2002. 19: 277-94

12. Espejo X, Tsunechiro MA, Osis MJD, Duarte GA, Bahamondese L, Sousa MH. AdeQuação do conhecimento sobre métodos

anticoncepcionais entre mulheres de Campinas, São Paulo. Rev Saúde Pública 2003; 37(5): 583-90.

13. Aleixo RPT. Perfil psicossocial e sexual da adolescente grávida atendida no pré- natal da maternidade Alexander Fleming uma perspectiva multidisciplinar [dissertação]. Rio de Janeiro: Universidade Gama Filho; 1998.

14. Castro CR. "Aconteceu, aí eu deixei vir": A representação social da gravidez em meninas de rua [dissertação]. Rio de Janeiro : Universidade do Estado do Rio de Janeiro; 1998.

15. Davim RMB. A prática da contracepção: causas de abandono na utilização de métodos contraceptivos por adolescentes [dissertação]. João Pessoa: Universidade Federal da Paraíba; 1998.

16. Cavalcanti SMOC. Fatores associados ao uso de anticoncepcionais na adolescência [dissertação]. Recife: Instituto Materno Infantil de Pernambuco; 2000.

17. Romero KT. Características do desenvolvimento físico e dos conhecimentos sobre sexualidade, métodos contraceptivos e doenças sexualmente transmissíveis de adolescentes do sexo feminino [dissertação]. São Paulo: Universidade Federal de São Paulo; 2003.

18. Berquó E, organizador). Comportamento sexual da população brasileira e percepções do HIV/Aids. São Paulo: Centro Brasileiro de Análise e Planejamento, Programa Nacional de DST/Aids. Brasília: Ministério da Saúde; 2000.

19. Almeida MC, Aquino EM, Gaffikin L, Magnani RJ. Contraceptive use among adolescents at public schools in Brazil. Rev Saúde Pública 2003; 37: 566-75.

20. Camarano AA. Fecundidade e anticoncepção da população jovem. In: Bereuó E, organizador. Jovens acontecendo na trilha das políticas públicas. Brasília: Comissão Nacional de População e Desenvolvimento; 1998. p. 109-33.

21. Béria J. Ficar, transar. A sexualidade do adolescente em tempos de AIDS. Porto Alegre: Tomo Editorial; 1998.

22. Castro MG, Abramovay M, Silva LB. Juventudes e sexualidade. Brasília: UNESCO Brasil; 2004.

23. Centro Brasileiro de Análise e Planejamento. Comportamento 
sexual da população brasileira e percepções do HIV/AIDS. Brasília: Ministério da Saúde; 2000.

24. Romero MI, Maddaleno M, Silber TI, Munist M. Salud reproductiva. In: Silber TJ, Munist MM, Maddaleno M, Ojeda ENS, organizadores. Manual de medicina de la adolescencia. Washington: Organización Panamericana de Salud; 1991. p. 473-82.

25. Pirotta KCM. Não há guarda chuva contra o amor: estudo do comportamento reprodutivo e de seu universo simbólico entre jovens universitários da USP [tese]. São Paulo: Universidade de São Paulo; 2002.

26. Costa MPSSM. Conhecimento, atitude e prática sobre métodos contraceptivos entre adolescentes com antecedentes reprodutivos em Teresina/PI [dissertação]. Teresina: Universidade Federal do Piauí; 2006.
27. Araújo TME. Vacinação Infantil: conhecimentos, atitudes e práticas da população da Área Norte/Centro de Teresina/PI [tese]. Rio de Janeiro: Universidade Federal do Rio de Janeiro; 2005.

28. Schor N, Ferreira AF, Machado VL, França AP, Pirotta K CM, Alvarenga AT. Mulher e anticoncepção: conhecimento e uso de métodos anticoncepcionais. Cad Saúde Pública 2000; I6(2): 377-84.

29. Araújo RT. Sentimentos, significados e aprendizagens de adolescentes grávidas em serviços de pré-natal buscando subsídios para a integralidade das ações [dissertação]. Rio de Janeiro: Universidade do Rio de Janeiro; 1998.

30. Rossato ARS. O significado da maternidade na adolescência [dissertação]. Porto Alegre: Pontifícia Universidade Católica do Rio Grande do Sul; 200I . 